\title{
Emerging Enteroviruses Causing Hand, Foot and Mouth Disease, China, 2010-2016
}

\author{
Yu Li, ${ }^{1}$ Zhaorui Chang, ${ }^{1}$ Peng Wu, ${ }^{1}$ \\ Qiaohong Liao, Fengfeng Liu, Yaming Zheng, \\ Li Luo, Yonghong Zhou, Qi Chen, \\ Shuanbao Yu, Chun Guo, Zhenhua Chen, \\ Lu Long, Shanlu Zhao, Bingyi Yang, \\ Hongjie Yu, ${ }^{2}$ Benjamin J. Cowling
}

Coxsackievirus A6 emerged as one of the predominant causative agents of hand, foot and mouth disease epidemics in many provinces of China in 2013 and 2015. This virus strain accounted for $25.9 \%$ of mild and $15.2 \%$ of severe cases in 2013 and $25.8 \%$ of mild and $16.9 \%$ of severe cases in 2015.

$\mathrm{H}$ and, foot and mouth disease (HFMD) is a common childhood infectious disease caused by enteroviruses (1). In China, HFMD cases must be reported to the Notifiable Infectious Diseases Reporting Information System. Apart from clinical and demographic information, case notifications also include etiologic results, if available, classified into 3 categories: enterovirus A71 (EV-A71), coxsackievirus (CV) A16, and other enteroviruses. However, not all cases have etiologic results, the Notifiable Infectious Diseases Reporting Information System (NIDRIS) does not indicate cases that tested negative for enteroviruses, and testing methods vary among hospitals (2). To capture more information on the etiologic spectrum of HFMD in China, a laboratory surveillance network has been established in provincial-level centers for disease control and prevention (CDCs). EV-A71 and CV-A16 were previously believed to be the main causative viruses for HFMD in Asia, but several studies have suggested that other enteroviruses appear

Author affiliations: Chinese Center for Disease Control and Prevention. Beijing, China (Y. Li, Z. Chang, Q. Liao, F. Liu, Y. Zheng, L. Luo, S. Yu, H. Yu); The University of Hong Kong, Hong Kong, China (Y. Li, P. Wu, B. Yang, B.J. Cowling); Fudan University, Shanghai, China (Y. Zhou, H. Yu); Hubei Center for Disease Prevention and Control, Wuhan, China (Q. Chen); Huazhong University of Science and Technology, Wuhan, China (C. Guo); Chengdu Center for Disease Prevention and Control, Chengdu, China (Z. Chen); Sichuan University, Chengdu, China (L. Long); Hunan Center for Disease Prevention and Control, Changsha, China (S. Zhao)

DOI: https://doi.org/10.3201/eid2410.171953 to be increasing since 2008 (3-9). Nevertheless, these past studies in China could not provide an overview at the national level because of limitations in geographic locations or study settings; furthermore, none of them systematically examined proportions of specific enteroviruses testing positive among tested HFMD cases. We analyzed data from this laboratory network to examine causative pathogens of HFMD cases and epidemiologic differences associated with various pathogens.

\section{The Study}

Since June 2009, clinical specimens must be collected from all severe HFMD cases, and the first 5 reported mild cases (case classification criteria in the online Technical Appendix, https://wwwnc.cdc.gov/EID/article/24/10/171953-Techapp1.pdf) in each county of China every month and are tested for enteroviruses at local CDCs using PCR (online Technical Appendix) (10). Test results are characterized as negative for enterovirus or positive for EV-A71, CV-A16, or other enteroviruses. For specimens testing positive for other enteroviruses, further serotyping is not conducted as a routine practice, but some local CDCs with more laboratory capacity may select a subset of these specimens to test on the serotype at their own discretion, especially when the proportion of other enteroviruses detected was relatively high.

We collected individual laboratory data during January 2010-December 2016 from 23 provincial CDCs (online Technical Appendix Figure 1). In these provinces, HFMD case notifications accounted for $88.4 \%$ of HFMD cases notified in China overall. We analyzed virus serotypes in combination with sex, age, and clinical severity of each case. The dataset includes 693,580 individual illness episodes in the 23 provinces; 7,632-59,507 (median 31,317) episodes per province were reported. Clinical samples were collected from each illness onset, including throat swabs $(374,685 ; 54.0 \%)$, feces $(153,947 ; 22.2 \%)$, rectal swabs $(129,837 ; 18.7 \%)$, and other specimens $(35,111 ; 5.1 \%)$ such as vesicular or cerebrospinal fluid.

Weekly proportions of positive enteroviruses (1 enterovirus-negative specimens divided by all specimens collected for testing) were generally lower in mild cases

\footnotetext{
${ }^{1}$ These authors contributed equally to this article.

${ }^{2}$ Current affiliation: Fudan University School of Public Health, Shanghai, China.
} 

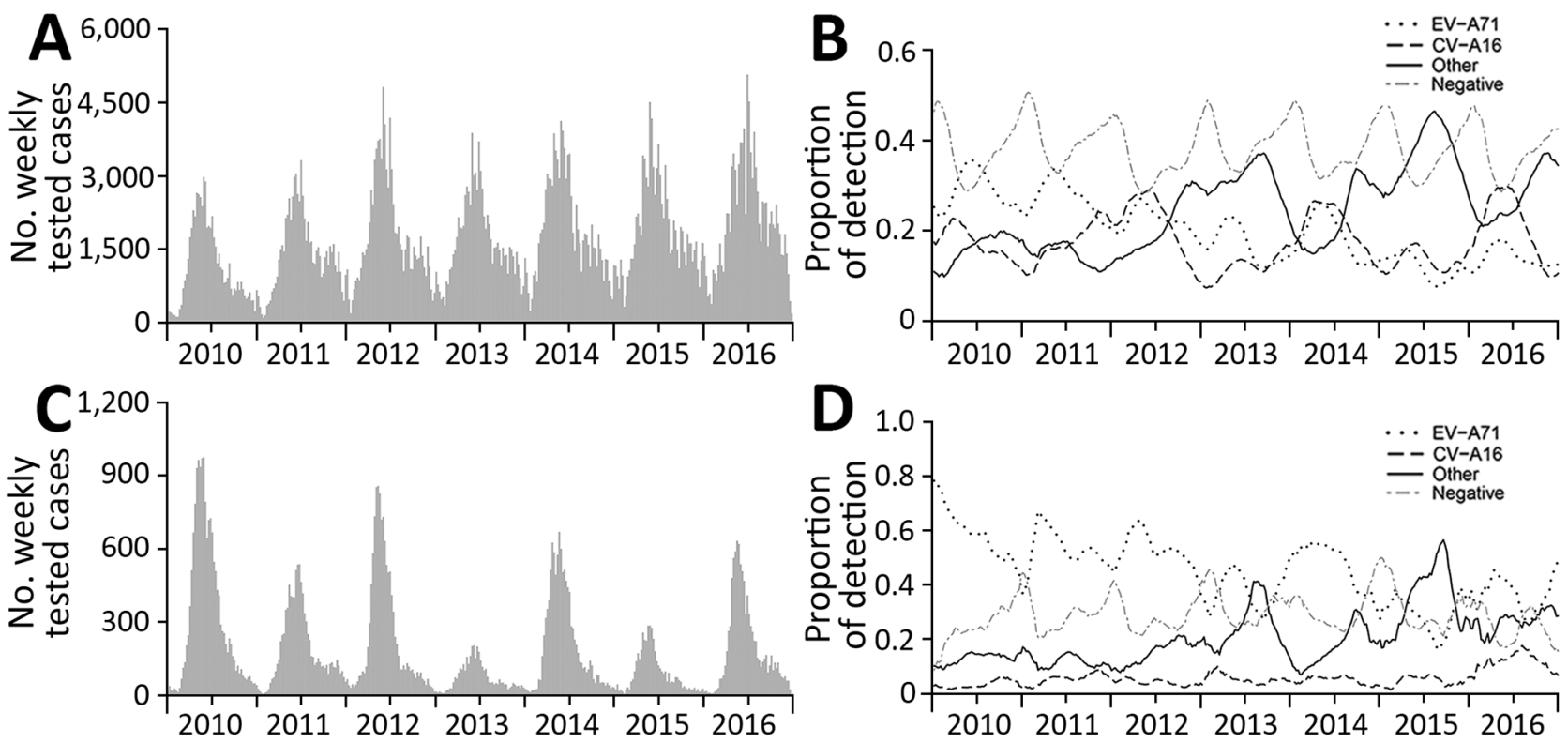

Figure 1. Weekly proportions of enteroviruses detection by serotype among hand, foot and mouth disease cases, January $2010-$ December 2016, China: A) number of tested mild cases; B) proportions of serotypes among mild cases C) number of tested severe cases; D) proportions of serotypes among severe cases.

\begin{tabular}{|c|c|c|c|c|}
\hline \multirow[b]{2}{*}{ Test result } & \multicolumn{2}{|c|}{2013, no. (\%) } & \multicolumn{2}{|c|}{2015, no. (\%) } \\
\hline & Mild, $\mathrm{n}=87,226$ & Severe, $\mathrm{n}=3,837$ & Mild, $n=99,461$ & Severe, $\mathrm{n}=4,712$ \\
\hline EV negative & $32,801(37.60)$ & $1,100(28.67)$ & $35,167(35.36)$ & $1,273(27.02)$ \\
\hline EV-A71 & 15,503 (17.77) & $1,557(40.58)$ & $11,800(11.86)$ & 1,352 (28.69) \\
\hline CV-A16 & 10,811 (12.39) & 197 (5.13) & 13,959 (14.03) & 265 (5.62) \\
\hline Other enteroviruses & $28,111(32.23)$ & $983(25.62)$ & $38,535(38.74)$ & $1,822(38.67)$ \\
\hline \multicolumn{5}{|c|}{ Further serotyping of other enteroviruses } \\
\hline Total & Mild, $n=3,260 \dagger$ & Severe, $n=42 \ddagger$ & Mild, $n=2,474 \S$ & Severe, $n=71 \pi$ \\
\hline CV-A6 & $2,620(80.37)$ & $28(66.67)$ & $1,471(59.46)$ & $31(43.70)$ \\
\hline CV-A10 & $176(5.40)$ & $4(9.52)$ & $104(4.20)$ & \# \\
\hline CV-A2 & $22(0.67)$ & $1(2.38)$ & $5(0.20)$ & \# \\
\hline CV-A5 & $9(0.28)$ & $1(2.38)$ & $2(0.08)$ & \# \\
\hline CV-A4 & $2(0.06)$ & \# & $9(0.36)$ & \# \\
\hline ECV-6 & $9(0.28)$ & \# & \# & \# \\
\hline CV-B4 & $13(0.4)$ & \# & \# & \# \\
\hline ECV-25 & $8(0.25)$ & \# & \# & \# \\
\hline CV-A12 & $5(0.15)$ & \# & \# & \# \\
\hline CV-B5 & $5(0.15)$ & \# & \# & \# \\
\hline CV-B2 & $4(0.12)$ & \# & $1(0.04)$ & \# \\
\hline ECV-7 & $4(0.12)$ & \# & \# & \# \\
\hline ECV-9 & $3(0.09)$ & \# & \# & \# \\
\hline CV-A8 & $1(0.03)$ & \# & $3(0.12)$ & \# \\
\hline CV-A14 & $2(0.06)$ & \# & \# & \# \\
\hline CV-A21 & $2(0.06)$ & \# & \# & \# \\
\hline ECV-12 & $2(0.06)$ & \# & \# & \# \\
\hline CV-B1 & $2(0.06)$ & \# & \# & \# \\
\hline ECV-30 & $1(0.03)$ & \# & $1(0.04)$ & \# \\
\hline Other & $6(0.18)^{\star *}$ & \# & \# & $\#$ \\
\hline Untyped & 364 (11.37) & $8(19.05)$ & 878 (35.49) & $40(56.34)$ \\
\hline
\end{tabular}

${ }^{*} \mathrm{CV}$, coxsackievirus; ECV, echovirus; EV, enterovirus.

†Results are based on data from Beijing, Fujian, Guangdong, Guangxi, Heilongjiang, Henan, Hubei, Jiangsu, Jilin, Shanxi, Sichuan, Shandong, Tianjin, Xinjiang, and Yunnan provinces.

tResults are based on data from Fujian, Guangdong, Guangxi, Henan, and Shanxi provinces.

§Results are based on data from Beijing, Fujian, Guangxi, Heilongjiang, Jiangsu, Sichuan, Tianjin, and Zhejiang provinces.

TResults are based on data from Guangdong and Jiangsu provinces.

\#The serotypes were not detected, but might be included in the untyped specimens because of laboratory capacity restrictions

${ }^{* *}$ Other serotypes include CV-A20, CV-A24, ECV-1, ECV-96, Polio1, and Polio2, with 1 of each serotype detected. 
(median $62.4 \%$, range $42.0 \%-74.0 \%$ ) than in severe cases (median $73.1 \%$, range $27.3 \%-100 \%$ ) and showed seasonal variations: peaks in April-May and low levels in December-January (Figure 1). The highest weekly proportion of EV-A71 detections among mild cases was $37.7 \%$ in 2010 ; a decreasing trend was observed thereafter. EV-A71 vaccine probably had little effect on the change in EV-A71 detections because it was not available until March 2016 and was not included in the routine vaccination program. In contrast, weekly proportions of detection of other enteroviruses generally increased with time, reaching a maximum of $48.4 \%$ in 2015 . The proportion of cases positive for CVA16 was relatively stable at $\approx 20 \%$ across the years, following a similar temporal trend to that of EV-A71. However, detections of different serotypes of enteroviruses generally demonstrated a similar temporal pattern among severe cases as among mild cases, except that the proportion of $\mathrm{CV}$-A16 was relatively low, fluctuating at $\approx 5 \%$ across the period (Figure 1). Proportions of detection generally declined with age for other enteroviruses, whereas EV-A71 and CV-A16 showed an increasing trend with age, particularly in mild cases (online Technical Appendix Figure 2).

EV-A71 and CV-A16 predominated in 2010-2012, 2014, and 2016, but other enteroviruses were predominant in 2013 and 2015. In those 2 years, further serotyping on other enteroviruses was widely conducted (Table). In 2013 , a total of 3,260 ( $11.6 \%$ of 28,111 specimens positive for other enteroviruses) specimens collected from mild cases and 42 (4.3\% of 983$)$ specimens collected from severe cases underwent further serotyping; in 2015, a total of $2,474(6.4 \%$ of 38,535$)$ specimens collected from mild cases and $71(3.9 \%$ of 1,822$)$ specimens collected from severe cases underwent further serotyping. The serotyping results showed that, of mild cases infected with other enteroviruses, $80 \%$ in 2013 and 59\% in 2015 were infected with CV-A6; for severe cases, 67\% in 2013 and $44 \%$ in 2015 were infected with CV-A6. By multiplying the proportion of other enteroviruses by the proportion of CV-A6 among other enteroviruses, we estimated that CV-A6 accounted for $25.9 \%$ of mild cases and $15.2 \%$ of severe cases in 2013 and $25.8 \%$ of mild cases and $16.9 \%$ of severe cases in 2015. This result at the national level supports regional and subregional studies in China (3-9), suggesting that $\mathrm{CV}-\mathrm{A} 6$ emerged as a main causative agent of HFMD in 2013 and 2015, but detections of CV-A6 were still low in some provinces of southwestern and northeastern China (Figure 2; online Technical Appendix Figure 3). D3 is the predominant subgenotype for CV-A6 (11). During the same period, CV-A10 accounted for $4.2 \%-9.5 \%$ of other enteroviruses. Serotypes other than CV-A6 and CVA10, including CV-A2, CV-A5, CV-A4, CV-B4, echovirus 6 and -25 , and others, accounted for a small proportion $(0.03 \%-2.4 \%)$ of specimens tested for further serotyping.
These rare serotypes could possibly become prevalent in the future because of accumulative immunity to the prevalent enteroviruses, the potential replacement effect induced by vaccination programs against predominant enteroviruses, or both. One limitation of serotyping results of other enteroviruses is that they tend to reflect those of areas with more intensive HFMD transmission, because local CDCs generally select areas where the most cases are detected for further serotyping.

We found that detection proportions of other enteroviruses were generally negatively associated with that of EV-A71 in mild and severe cases (Pearson correlation coefficient -0.73 for mild cases and -0.70 for severe cases) and CV-A16 in mild cases (Pearson correlation coefficient -0.52) (Figure 1). This result might indicate competitive interactions between other enteroviruses and EV-A71 or CV-A16, which should be considered when evaluating the effect of introducing a new enterovirus vaccine, especially when the proportion of other enteroviruses is increasing. The epidemiologic modeling study suggested that crossprotection between EV-A71 and CV-A16 exists for nearly 7 weeks, on average, in the context of natural infections (12). However, vaccine trials reported that monovalent EV-A71 vaccine failed to protect against CV-A16-associated HFMD (13). Similarly, whether cross protection exists between EV-A71 and other enteroviruses, such as CV-A6 and CV-A10, remains poorly understood to date, although limited studies have been more indicative of a lack of cross protection between EV-A71 and coxsackieviruses including CV-A6 $(14,15)$.

\section{Conclusions}

Data from national laboratory network surveillance of HFMD in China show that detection of enteroviruses other than EV-71 and CV-A16 has been increasing in both mild and severe cases and that CV-A6 has been emerging as another predominant serotype recently, but not in every province. Serotyping of individual enteroviruses apart from currently tested EV-71 and CV-A16 is suggested for routine virologic surveillance. Further studies may be needed to investigate potential cross immunity between EV-A71 and other enteroviruses such as CV-A6, CV-A10, and others.

\section{Acknowledgments}

We thank county-, prefecture-, and province-level CDCs in the 23 provinces for their kind support and assistance in collecting data.

This study was funded by the National Science Fund for Distinguished Young Scholars (grant no. 81525023), the US National Institutes of Health (Comprehensive International Program for Research on AIDS grant no. U19 AI51915), Chinese Center for Disease Control and Prevention's Key 


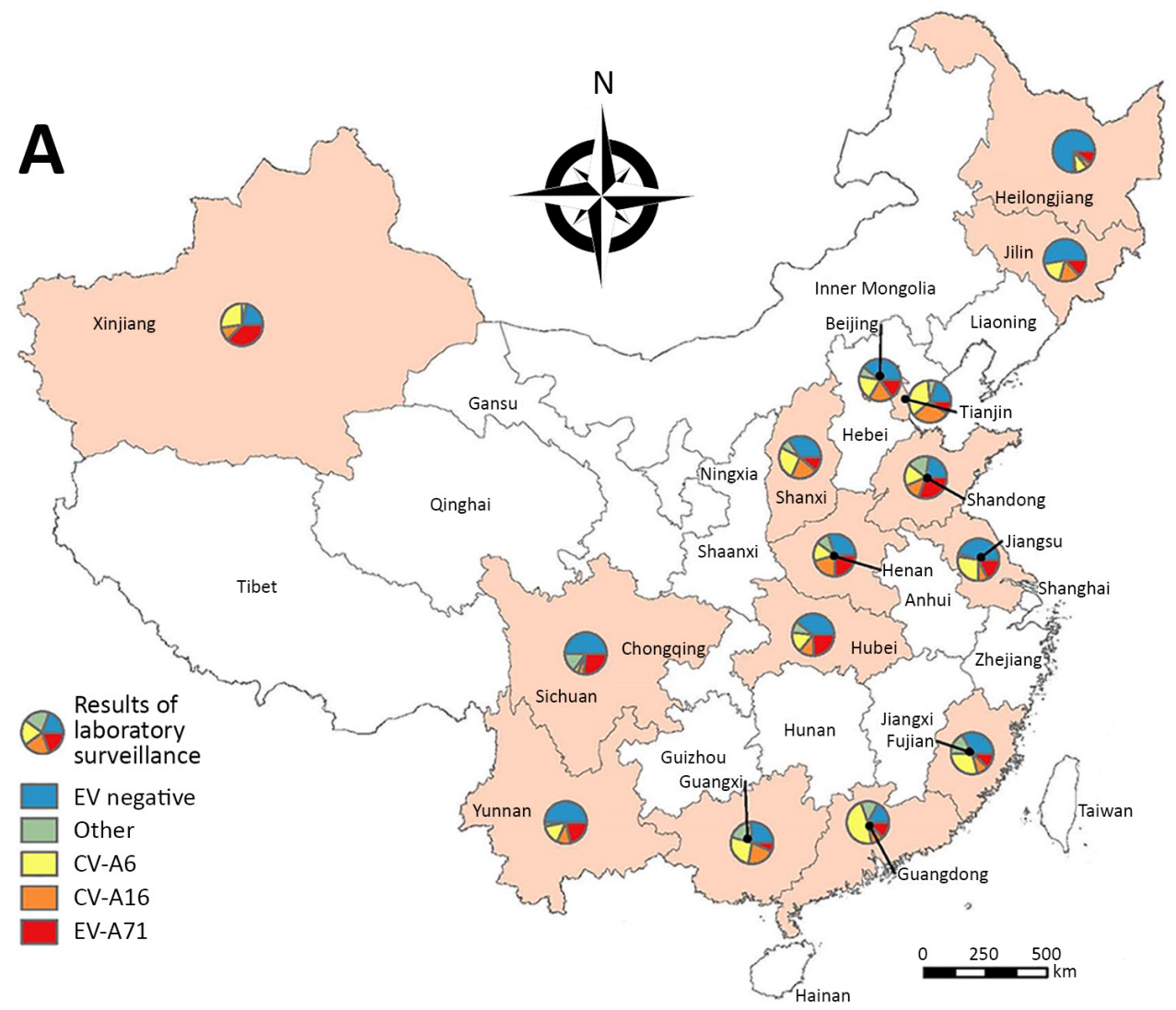

Figure 2. Estimated yearly detection proportions of CV-A6, EV-A71, CV-A16, and other enteroviruses among mild hand, foot and mouth disease cases by province in China: A) 2013; B) 2015.

CV, coxsackievirus; $E V$, enterovirus.

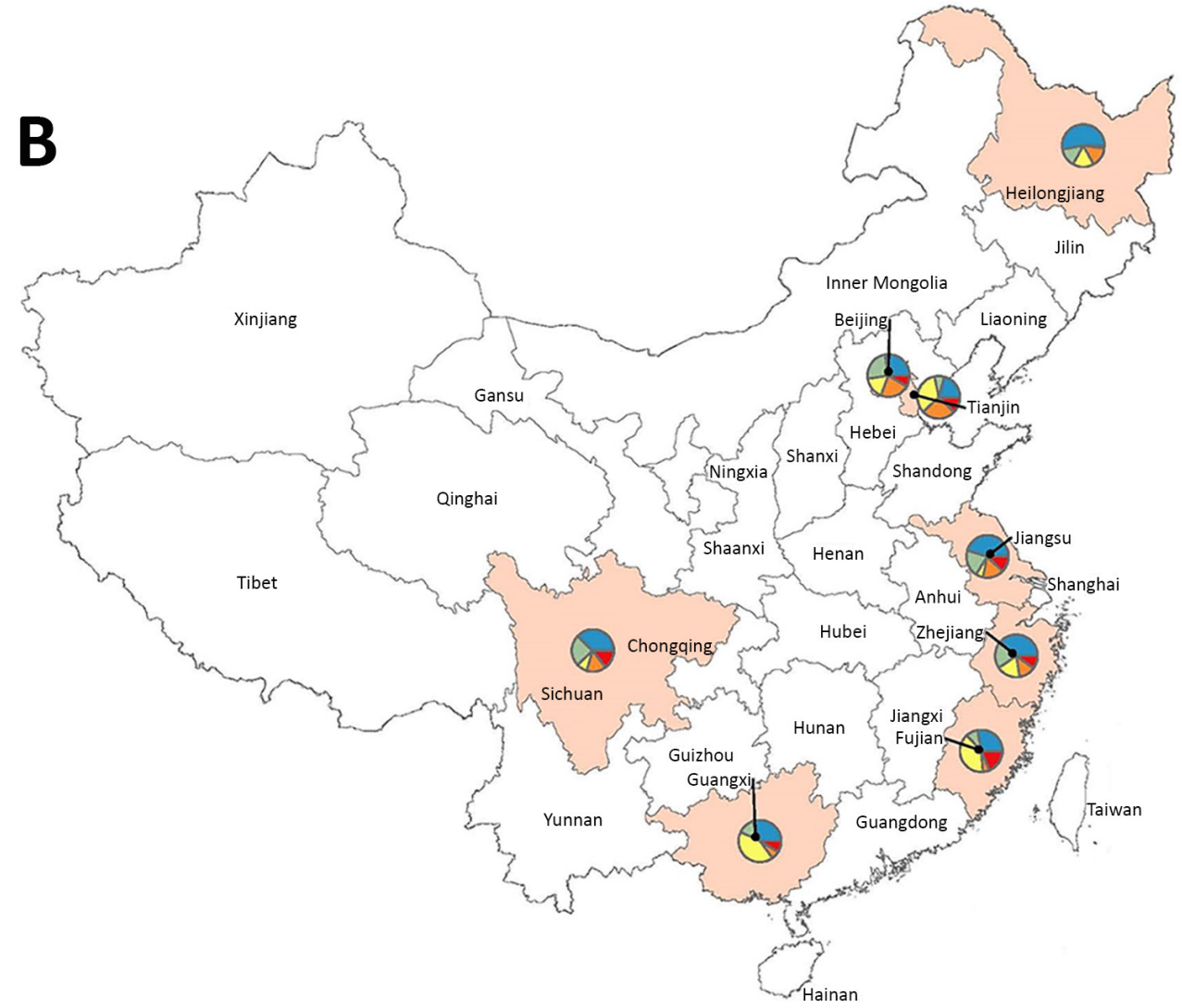


Laboratory of Surveillance and Early Warning on Infectious Disease, the Harvard Center for Communicable Disease Dynamics from the National Institute of General Medical Sciences (grant no. U54 GM088558), the Research Grants Council of the Hong Kong Special Administrative Region, China (project no. T11-705/14N), the National Natural Science Foundation of China (grant no. 81473031), the Natural Science Foundation of Shanghai (grant no. 14ZR1444500), the Li Ka Shing Oxford Global Health Programme (grant no. B9RST00-B900.57), and the TOTAL Foundation (grant no. 2015-099).

\section{About the Author}

Dr. Li is a doctoral candidate at the School of Public Health, the University of Hong Kong. His primary research interests include epidemiology and transmission dynamics of zoonotic and vectorborne diseases, and hand, foot and mouth disease.

\section{References}

1. Aswathyraj S, Arunkumar G, Alidjinou EK, Hober D. Hand, foot and mouth disease (HFMD): emerging epidemiology and the need for a vaccine strategy. Med Microbiol Immunol (Berl). 2016;205:397-407. http://dx.doi.org/10.1007/s00430-016-0465-y

2. Yang B, Liu F, Liao Q, Wu P, Chang Z, Huang J, et al. Epidemiology of hand, foot and mouth disease in China, 2008 to 2015 prior to the introduction of EV-A71 vaccine. Euro Surveill. 2017;22. http://dx.doi.org/10.2807/1560-7917. ES.2017.22.50.16-00824

3. Hongyan G, Chengjie M, Qiaozhi Y, Wenhao H, Juan L, Lin P, et al. Hand, foot and mouth disease caused by coxsackievirus A6, Beijing, 2013. Pediatr Infect Dis J. 2014;33:1302-3. http://dx.doi.org/10.1097/INF.0000000000000467

4. Li JS, Dong XG, Qin M, Feng HR, Yang JY, Li RX, et al. Outbreak of hand, foot, and mouth disease caused by coxsackievirus A6 in a Juku in Fengtai District, Beijing, China, 2015. Springerplus. 2016;5:1650. http://dx.doi.org/10.1186/s40064-016-3307-x

5. Xia Y, Shan J, Ji H, Zhang J, Yang H, Shen Q, et al. Study of the epidemiology and etiological characteristics of hand, foot, and mouth disease in Suzhou City, East China, 2011-2014. Arch Virol. 2016;161:1933-43. http://dx.doi.org/10.1007/s00705-016-2878-8

6. Zeng H, Lu J, Zheng H, Yi L, Guo X, Liu L, et al. The epidemiological study of coxsackievirus A6 revealing hand, foot and mouth disease epidemic patterns in Guangdong, China. Sci Rep. 2015;5:10550. http://dx.doi.org/10.1038/srep10550
7. Weng Y, Chen W, He W, Huang M, Zhu Y, Yan Y. Serotyping and genetic characterization of hand, foot, and mouth disease (HFMD) -associated enteroviruses of non-EV71 and nonCVA16 circulating in Fujian, China, 2011-2015. Med Sci Monit. 2017;23:2508-18. http://dx.doi.org/10.12659/MSM.901364

8. Li Y, Bao H, Zhang X, Zhai M, Bao X, Wang D, et al. Epidemiological and genetic analysis concerning the non-enterovirus 71 and non-coxsackievirus A16 causative agents related to hand, foot and mouth disease in Anyang city, Henan Province, China, from 2011 to 2015. J Med Virol. 2017;89: 1749-58. http://dx.doi.org/10.1002/jmv.24847

9. Bian L, Wang Y, Yao X, Mao Q, Xu M, Liang Z. Coxsackievirus A6: a new emerging pathogen causing hand, foot and mouth disease outbreaks worldwide. Expert Rev Anti Infect Ther. 2015; 13:1061-71. http://dx.doi.org/10.1586/14787210.2015.1058156

10. Xing W, Liao Q, Viboud C, Zhang J, Sun J, Wu JT, et al. Hand, foot, and mouth disease in China, 2008-12: an epidemiological study. Lancet Infect Dis. 2014;14:308-18. http://dx.doi.org/10.1016/S1473-3099(13)70342-6

11. Song Y, Zhang Y, Ji T, Gu X, Yang Q, Zhu S, et al. Persistent circulation of coxsackievirus A6 of genotype D3 in mainland of China between 2008 and 2015. Sci Rep. 2017;7:5491. http://dx.doi.org/10.1038/s41598-017-05618-0

12. Takahashi S, Liao Q, Van Boeckel TP, Xing W, Sun J, Hsiao VY, et al. Hand, foot, and mouth disease in China: modeling epidemic dynamics of enterovirus serotypes and implications for vaccination. PLoS Med. 2016;13:e1001958. http://dx.doi.org/10.1371/ journal.pmed.1001958

13. Zhu FC, Meng FY, Li JX, Li XL, Mao QY, Tao H, et al. Efficacy, safety, and immunology of an inactivated alum-adjuvant enterovirus 71 vaccine in children in China: a multicentre, randomised, double-blind, placebo-controlled, phase 3 trial. Lancet. 2013;381:2024-32. http://dx.doi.org/10.1016/ S0140-6736(13)61049-1

14. Liu CC, Guo MS, Wu SR, Lin HY, Yang YT, Liu WC, et al. Immunological and biochemical characterizations of coxsackievirus A6 and A10 viral particles. Antiviral Res. 2016;129:58-66. http://dx.doi.org/10.1016/j.antiviral.2016.02.008

15. Lin Y, Wen K, Pan Y, Wang Y, Che X, Wang B. Cross-reactivity of anti-EV71 IgM and neutralizing antibody in series sera of patients infected with enterovirus 71 and coxsackievirus A 16. J Immunoassay Immunochem. 2011;32:233-43. http://dx.doi.org/ $10.1080 / 15321819.2011 .559297$

Address for correspondence: Hongjie Yu, School of Public Health,

Fudan University, Key Laboratory of Public Health Safety, Ministry of Education, 130 Dong'an Rd, Xuhui District, Shanghai 200032, China; email: cfetpyhj@vip.sina.com 